Для того чтобы обезопасить личную информацию, специалисты советуют повышать компьютерную грамотность и следовать нескольким важным требованиям.

1. Нельзя переходить по сомнительным ссылкам.

При переходе вы можете занести вредоносное ПО в свой компьютер, которое предоставим хакерам ваши данные.

Чтобы не подвергаться к риску, старайтесь заходить на сайты с хорошей репутацией.

2. Скачивание фальшивого антивирусного ПО.

Довольно каждый пользователь сталкивался с всплывающими предупреждениями о том, что ваш компьютер окажется под угрозой, если вы немедленно не скачаете бесплатное антивирусное ПО. Этого ни в коем случае нельзя делать, ведь один клик мыши по этой ссылке может заблокировать использование на компьютере защитных решений.

3. Использование слабых паролей.

Пароли могут быть легко угаданы злоумышленником, если не принять мер предосторожности. Ваш пароль должен содержать и цифры, и знаки препинания, и заглавные и строчные буквы. Избегайте использования каких-либо слов или комбинаций слов, имен и т.д. Лучше всего использовать пароль, каждый символ которого соответствует какому-то слову. Обычно следует иметь различные пароли для различных учетных записей, но не в таком количестве, чтобы вы не смогли запомнить их. [4]

4. Хранение всех своих паролей в системе.

Этого ни в коем случае нельзя делать, потому что если вашу систему взломают, то у злоумышленников будет доступ ко всем вашим паролям и они смогут проникнуть в другие ваши данные.

Для обеспечения защиты персональных данных следует предпринимать следующие меры.

1. Нужно зашифровать всю важную информацию.

2. Использовать антивирусные программы и вовремя их обновлять.

3. Не скачивать подозрительные приложения.

4. Использовать протокол HTTPS.

5. Ставить надежные пароли.

6. Включить двойную аутентификацию.

Надо понимать, что никто не застрахован от кибератак и утечки информации. Чтобы не попасться на взлом злоумышленников и обеспечить безопасность своих данных, необходимо придерживаться вышеизложенных элементарных мер предосторожности при работе с персональным компьютером и сети интернет. Нужно помнить, если вы стали жертвой мошенников, обязательно принимайте необходимые меры, иначе ваши личные сведения могут быть использованы в самых различных целях.

$$
\text { **** }
$$

1. Википедия https://ru.m.wikipedia.org/wiki/Угрозы_информационной_безопасности

2. Хакерские атаки https://ru.malwarebytes.com/hacker/

3. Белов Е. Б., Лось В. П. Основы информационной безопасности. М. : Горячая линия : Теле- ком, 2006.

4. Скембрей Д., Мак-Клар С. Секреты хакеров. Безопасность Microsoft Windows Server - Hacking Exposed Windows Server - М.: «Вильямс», 2007. - С. 512.

\title{
Вавилов В.А. \\ Асимптотический анализ RQ-системы с вызываемыми и возвратными заявками в случайной среде
}

Национальный исследовательский Томский государственньий университет (Россия, Томск)

doi: 10.18411/trnio-11-2021-37

\section{Аннотация}

B работе предложена математическая модель RQ-системы с вызываемыми и возвратными заявками в случайной среде, моделируемой цепью Маркова с непрерывным 
временем. Проведено исследование представленной модели методом асимптотического анализа в условиях возрастающей задержки заявок на орбите. Получено распределение вероятностей состояний прибора, асимптотическое среднее нормированного числа заявок на орбите, а также величины отклонения от среднего.

Ключевые слова: RQ-система, система с вызываемыми заявками, случайная среда, орбита, асимптотический анализ.

\section{Abstract}

The paper proposes a mathematical model of the retrial queue with two way communication, returnable requests in a random environment modeled by a Markov chain with continuous time. The presented model researched by the method of asymptotic analysis under conditions of increasing delay of applications in orbit. The probability distribution of the device states, the asymptotic average of the normalized number of applications in orbit, as well as the deviation from the average are obtained.

Keywords: retrial queue with two-way communication, random environment, orbit, asymptotic analysis.

\section{Введение}

Периодичность развития экономических систем требует от управления банков своевременного реагирования на изменения экономической конъюнктуры. Методы диагностики финансового состояния основаны на анализе большого объёма информации, при этом, алгоритмы вычисления показателей эффективности финансовой деятельности сложны и не всегда очевидны. Это приводит к тому, что результаты анализа не отражают в полной мере реальную ситуацию и недостаточно наглядно характеризуют устойчивость кредитной организации.

Математическое моделирование банковской деятельности позволяет выявить определённые закономерности и увеличить скорость управленческой реакции на изменения внешней среды. В силу стохастического характера изучаемых процессов, в данной работе представим деятельность банка в виде модели функционирования RQсистемы (retrial queue - системы с повторными вызовами) [1-2]. В рассматриваемой модели учтём работу call-центра - важного элемента структуры банковской организации, призванного рекламировать и продвигать банковские продукты и услуги, привлекать клиентов и сокращать так называемое время простоя. Немаловажным фактором успешной финансовой деятельности является наличие постоянных клиентов, поэтому учтём тот факт, что по завершении успешного обслуживания клиент может покинуть систему навсегда или через некоторый промежуток времени вернуться для повторного обслуживания. Например, выплативший кредит клиент может повторно обратиться за новым кредитом или другой банковской услугой или продуктом.

Кроме того, параметры функционирования таких систем обслуживания зависят от ряда факторов, характер влияния которых можно определить, как случайный [3]. Совокупность неконтролируемых внешних экономических, социальных и иных факторов называют случайной средой. Модель случайной среды может быть представлена однородной цепью Маркова с непрерывным временем [4-8], а в обобщённом случае полумарковским процессом [9-11].

Вследствие высокой сложности, получение точных аналитических выражений для вероятностно-временных характеристик рассматриваемых систем обслуживания затруднено или невозможно. По этой причине применяют асимптотические методы исследования [12-13]. Исследование в этом случае сводится к аппроксимации диффузионными процессами, отысканию коэффициентов переноса и диффузии.

B данной работе впервые представлена математическая модель RQ-системы с вызываемыми и возвратными заявками в случайной среде, моделируемой цепью Маркова 
с непрерывным временем. Проведён асимптотический анализ предложенной модели и получены основные вероятностные характеристики.

\section{1. Математическая модель}

Рассмотрим RQ-систему, на вход которой поступает простейший с параметром $\lambda$ поток заявок (клиентов). Заявка, заставшая в момент поступления прибор свободным, начинает обслуживаться в течение экспоненциально распределённого промежутка времени с параметром $\mu_{1}$. Свободный прибор может вызывать для обслуживания дополнительные заявки из внешней среды с интенсивностью $\alpha$. Вызываемая заявка занимает прибор для обслуживания, длительность которого имеет экспоненциальное распределение с параметром $\mu_{2}$. По завершении обслуживания заявка с вероятностью $\beta$ переходит на орбиту или покидает систему с вероятностью $1-\beta$. Если в течение обслуживания одной заявки поступает другая, то поступившая заявка переходит на орбиту. Повторное обращение заявок к прибору из орбиты происходит после случайной задержки, продолжительность которой имеет экспоненциальное распределение с параметром $\gamma$. Количество заявок на орбите обозначим $i$. Таким образом, прибор RQсистемы может находиться в одном из трёх состояний: $k=0$, если он свободен; $k=1$, если он занят обслуживанием заявки; $k=2$, если на приборе реализуется обслуживание вызываемой заявки.

$\mathrm{RQ}$-система функционирует в случайной среде, моделируемой однородной цепью Маркова $s(t)$ [14] с конечным множеством состояний $s=1,2, \ldots, S$ и непрерывным временем, для которой заданы её инфинитезимальные характеристики $q_{s_{1} s_{2}}$. Очевидно, что:

$$
\sum_{s_{2}=1}^{S} q_{s_{1} s_{2}}=0, s_{1}=1,2, \ldots, S .
$$

Влияние случайной среды на функционирование RQ-системы определяется зависимостью интенсивностей $\mu_{1}$ и $\mu_{2}$ обслуживания заявок от состояний $s(t)=s$ случайной среды, то есть $\mu_{1}=\mu_{1}(s), \mu_{2}=\mu_{2}(s)$. Вероятности окончания обслуживания заявок на приборе за бесконечно малый промежуток времени $\Delta t$ соответственно равны: $\mu_{1}(s) \Delta t+o(\Delta t)$ и $\mu_{2}(s) \Delta t+o(\Delta t)$.

В силу свойств описанной модели случайный процесс $\{k(t), i(t), s(t)\}$ изменения во времени состояний $\{k(t), i(t)\}$ RQ-системы и состояний $\{s(t)\}$ случайной среды, является цепью Маркова с непрерывным временем.

Для распределения $P_{k}(i, s, t)=P(k(t)=k, i(t)=i, s(t)=s) \quad$ можно $\quad$ составить систему [15] дифференциальных уравнений Колмогорова:

$$
\begin{gathered}
\frac{\partial P_{0}(i, s, t)}{\partial t}+(\alpha+\lambda+i \gamma) P_{0}(i, s, t)=(1-\beta) \mu_{1}(s) P_{1}(i, s, t)+\beta \mu_{1}(s) P_{1}(i-1, s, t)+ \\
+(1-\beta) \mu_{2}(s) P_{2}(i, s, t)+\beta \mu_{2}(s) P_{2}(i-1, s, t)+\sum_{s_{1}=1}^{S} q_{s_{1} s} P_{0}\left(i, s_{1}, t\right), \\
\frac{\partial P_{1}(i, s, t)}{\partial t}+\left(\lambda+\mu_{1}(s)\right) P_{1}(i, s, t)=\lambda P_{0}(i, s, t)+(i+1) \gamma P_{0}(i+1, s, t)+ \\
+\lambda P_{1}(i-1, s, t)+\sum_{s_{1}=1}^{S} q_{s_{1} s} P_{1}\left(i, s_{1}, t\right),
\end{gathered}
$$




$$
\frac{\partial P_{2}(i, s, t)}{\partial t}+\left(\lambda+\mu_{2}(s)\right) P_{2}(i, s, t)=\alpha P_{0}(i, s, t)+\lambda P_{2}(i-1, s, t)+\sum_{s_{1}=1}^{S} q_{s_{1} s} P_{2}\left(i, s_{1}, t\right) .
$$

В любой момент времени должно выполняться условие нормировки: $\sum_{k=0}^{2} \sum_{i=0}^{\infty} \sum_{s=1}^{S} P_{k}(i, s, t)=1$

Систему (2) будем исследовать методом асимптотического анализа [13] в условиях большой задержки $\gamma \rightarrow 0$. Для этого обозначим: $\gamma=\varepsilon^{2}, \varepsilon^{2} t=\tau$ и покажем, что предельный процесс $x(\tau)=\lim _{\varepsilon \rightarrow 0}\left(\varepsilon^{2} i\left(\tau / \varepsilon^{2}\right)\right)$, имеющий смысл асимптотического среднего нормированного числа заявок на орбите, является детерминированной функцией. Также докажем, что процесс $y(\tau)=\lim _{\varepsilon \rightarrow 0}\left(\left(\varepsilon^{2} i\left(\tau / \varepsilon^{2}\right)-x(\tau)\right) / \varepsilon\right)$, характеризующий изменение величин отклонения нормированного числа заявок на орбите, является диффузионным процессом [14] авторегрессии.

Итак, в системе (1) выполним замены $\varepsilon^{2} i=x+\varepsilon y, P_{k}(i, s, t)=\varepsilon H_{k}(y, s, \tau, \varepsilon)$, получим:

$$
\begin{gathered}
\varepsilon^{2} \frac{\partial H_{0}(y, s, \tau, \varepsilon)}{\partial \tau}-\varepsilon x^{\prime}(\tau) \frac{\partial H_{0}(y, s, \tau, \varepsilon)}{\partial y}+(\alpha+\lambda+x+\varepsilon y) H_{0}(y, s, \tau, \varepsilon)= \\
=(1-\beta) \mu_{1}(s) H_{1}(y, s, \tau, \varepsilon)+\beta \mu_{1}(s) H_{1}(y-\varepsilon, s, \tau, \varepsilon)+ \\
+(1-\beta) \mu_{2}(s) H_{2}(y, s, \tau, \varepsilon)+\beta \mu_{2}(s) H_{2}(y-\varepsilon, s, \tau, \varepsilon)+\sum_{s_{1}=1}^{S} q_{s_{1} s} H_{0}\left(y, s_{1}, \tau, \varepsilon\right), \\
\varepsilon^{2} \frac{\partial H_{1}(y, s, \tau, \varepsilon)}{\partial \tau}-\varepsilon x^{\prime}(\tau) \frac{\partial H_{1}(y, s, \tau, \varepsilon)}{\partial y}+\left(\lambda+\mu_{1}(s)\right) H_{1}(y, s, \tau, \varepsilon)= \\
=\lambda H_{1}(y-\varepsilon, s, \tau, \varepsilon)+(x+\varepsilon(y+\varepsilon)) H_{0}(y+\varepsilon, s, \tau, \varepsilon)+ \\
\quad+\lambda H_{0}(y, s, \tau, \varepsilon)+\sum_{s_{1}=1}^{S} q_{s_{1} s} H_{1}\left(y, s_{1}, \tau, \varepsilon\right), \\
\varepsilon^{2} \frac{\partial H_{2}(y, s, \tau, \varepsilon)}{\partial \tau}-\varepsilon x^{\prime}(\tau) \frac{\partial H_{2}(y, s, \tau, \varepsilon)}{\partial y}+\left(\lambda+\mu_{2}(s)\right) H_{2}(y, s, \tau, \varepsilon)= \\
=\alpha H_{0}(y, s, \tau, \varepsilon)+\lambda H_{2}(y-\varepsilon, s, \tau, \varepsilon)+\sum_{s_{1}=1}^{S} q_{s_{1} s} H_{2}\left(y, s_{1}, \tau, \varepsilon\right) .
\end{gathered}
$$

Дальнейшие исследования будем проводить на основе системы (3).

\section{2. Асимптотические средние характеристики}

Под асимптотическими средними характеристиками, будем понимать распределение вероятностей $R_{k}(x)$ состояний $k$ канала и функцию $x=x(\tau)$.

В системе (3) перейдём к пределу при $\varepsilon \rightarrow 0$ и, полагая, что существуют конечные пределы

$$
\lim _{\varepsilon \rightarrow 0} H_{k}(y, s, \tau, \varepsilon)=H_{k}(y, s, \tau),
$$

получим систему:

$$
(\alpha+\lambda+x) H_{0}(y, s, \tau)=\mu_{1}(s) H_{1}(y, s, \tau)+\mu_{2}(s) H_{2}(y, s, \tau)+\sum_{s_{1}=1}^{S} q_{s_{1} s} H_{0}\left(y, s_{1}, \tau\right),
$$




$$
\begin{array}{r}
\mu_{1}(s) H_{1}(y, s, \tau)=(\lambda+x) H_{0}(y, s, \tau)+\sum_{s_{1}=1}^{S} q_{s_{1} s} H_{1}\left(y, s_{1}, \tau\right) \\
\mu_{2}(s) H_{2}(y, s, \tau)=\alpha H_{0}(y, s, \tau)+\sum_{s_{1}=1}^{S} q_{s_{1} s} H_{2}\left(y, s_{1}, \tau\right) .
\end{array}
$$

В силу однородности полученной системы (5) линейных алгебраических уравнений будем искать её решение $H_{k}(y, s, \tau)$ в виде:

$$
H_{k}(y, s, \tau)=Q_{k}(x, s) H(y, \tau),
$$

где $H(y, \tau)$ является мультипликативной составляющей решения однородной системы, имеет смысл плотности распределения вероятностей значений процесса $y(\tau)$, а $Q_{k}(x, s)$ - есть двумерное распределение вероятностей состояний $k$ прибора и состояний $s$ случайной среды при условии $x(\tau)=x$, как следует из (5), определяется системой

$$
\begin{gathered}
(\alpha+\lambda+x) Q_{0}(x, s)=\mu_{1}(s) Q_{1}(x, s)+\mu_{2}(s) Q_{2}(x, s)+\sum_{s_{1}=1}^{S} q_{s_{1} s} Q_{0}\left(x, s_{1}\right), \\
\mu_{1}(s) Q_{1}(x, s)=(\lambda+x) Q_{0}(x, s)+\sum_{s_{1}=1}^{S} q_{s_{1} s} Q_{1}\left(x, s_{1}\right), \\
\mu_{2}(s) Q_{2}(x, s)=\alpha Q_{0}(x, s)+\sum_{s_{1}=1}^{S} q_{s_{1} s} Q_{2}\left(x, s_{1}\right)
\end{gathered}
$$

и условием нормировки:

$$
\sum_{k=0}^{2} \sum_{s=1}^{S} Q_{k}(x, s)=1
$$

Обозначим:

$$
\sum_{k=0}^{2} Q_{k}(x, s)=r(s), \quad \sum_{s=1}^{S} Q_{k}(x, s)=R_{k}(x)
$$

здесь $R_{k}(x)$ и $r(s)$ - маргинальные распределения вероятностей соответственно состояний $k$ прибора и $s$ - случайной среды. Для них также должны выполняться условия нормировки:

$$
\sum_{s=1}^{S} r(s)=1, \quad \sum_{k=0}^{2} R_{k}(x)=1 .
$$

Сложим по $k$ уравнения системы (7) и с учётом (9) получим систему $S$ уравнений вида: $\sum_{s_{1}=1}^{S} r\left(s_{1}\right) q_{s_{1} s}=0, s=1,2, \ldots, S$, которая совместно с условием нормировки (10) определяет стационарное распределение вероятностей $r(s)$ состояний цепи Маркова $s(t)$

Сложим по $s$ уравнения системы (7), учтём (9) и (1), обозначим:

$$
\sum_{s=1}^{S} \mu_{1}(s) Q_{1}(x, s)=\psi R_{1}(x), \quad \sum_{s=1}^{S} \mu_{2}(s) Q_{2}(x, s)=\varphi R_{2}(x),
$$

тогда система (7) примет вид:

$$
(\alpha+\lambda+x) R_{0}(x)=\psi R_{1}(x)+\varphi R_{2}(x), \quad \psi R_{1}(x)=(\lambda+x) R_{0}(x),
$$




$$
\varphi R_{2}(x)=\alpha R_{0}(x) .
$$

Система (12) совместно с условием нормировки (10) даёт решение:

$$
R_{0}(x)=\psi \varphi / G(x), \quad R_{1}(x)=(\lambda+x) \varphi / G(x), \quad R_{2}(x)=\alpha \psi(x) / G(x),
$$
где $G(x)=\alpha \psi+(\lambda+x) \varphi+\psi \varphi$.

Далее покажем, что $x=x(\tau)$ является детерминированной функцией.

В системе (3) функции $H_{k}(y \pm \varepsilon, s, \tau, \varepsilon)$ разложим в ряд по приращениям аргумента $y$ с точностью до $o(\varepsilon)$, получим:

$$
\begin{gathered}
-\varepsilon x^{\prime}(\tau) \frac{\partial H_{0}(y, s, \tau, \varepsilon)}{\partial y}+(\alpha+\lambda+x+\varepsilon y) H_{0}(y, s, \tau, \varepsilon)= \\
=\mu_{1}(s) H_{1}(y, s, \tau, \varepsilon)+\mu_{2}(s) H_{2}(y, s, \tau, \varepsilon)- \\
-\beta \mu_{1}(s) \varepsilon \frac{\partial H_{1}(y, s, \tau, \varepsilon)}{\partial y}-\beta \mu_{2}(s) \varepsilon \frac{\partial H_{2}(y, s, \tau, \varepsilon)}{\partial y}+\sum_{s_{1}=1}^{S} q_{s_{1} s} H_{0}\left(y, s_{1}, \tau, \varepsilon\right)+o(\varepsilon), \\
-\varepsilon x^{\prime}(\tau) \frac{\partial H_{1}(y, s, \tau, \varepsilon)}{\partial y}+\mu_{1}(s) H_{1}(y, s, \tau, \varepsilon)=-\varepsilon \lambda \frac{\partial H_{1}(y, s, \tau, \varepsilon)}{\partial y}+ \\
+(x+\varepsilon y) H_{0}(y, s, \tau, \varepsilon)+\varepsilon x \frac{\partial H_{0}(y, s, \tau, \varepsilon)}{\partial y}+\sum_{s_{1}=1}^{S} q_{s_{1} s} H_{1}\left(y, s_{1}, \tau, \varepsilon\right)+o(\varepsilon) \\
-\varepsilon x^{\prime}(\tau) \frac{\partial H_{2}(y, s, \tau, \varepsilon)}{\partial y}+\mu_{2}(s) H_{2}(y, s, \tau, \varepsilon)=\alpha H_{0}(y, s, \tau, \varepsilon)- \\
-\varepsilon \lambda \frac{\partial H_{2}(y, s, \tau, \varepsilon)}{\partial y}+\sum_{s_{1}=1}^{S} q_{s_{1} s} H_{2}\left(y, s_{1}, \tau, \varepsilon\right)+o(\varepsilon) .
\end{gathered}
$$

Уравнения (14) просуммируем по $k$ и по $s$, и учитывая (1), запишем:

$$
\begin{gathered}
-\varepsilon x^{\prime}(\tau) \frac{\partial}{\partial y}\left\{\sum_{k=0}^{2} \sum_{s=1}^{S} H_{k}(y, s, \tau, \varepsilon)\right\}=\varepsilon \frac{\partial}{\partial y}\left\{x \sum_{s=1}^{S} H_{0}(y, s, \tau, \varepsilon)-\right. \\
\left.-\sum_{s=1}^{S}\left(\lambda+\beta \mu_{1}(s)\right) H_{1}(y, s, \tau, \varepsilon)-\sum_{s=1}^{S}\left(\lambda+\beta \mu_{2}(s)\right) H_{2}(y, s, \tau, \varepsilon)\right\}+o(\varepsilon) .
\end{gathered}
$$

Поделим на $\varepsilon$ обе части полученного уравнения, выполним предельный переход (4), учтём равенство (6), условие нормировки (8), обозначение (9), получим, что функция $x=x(\tau)$ - есть решение обыкновенного дифференциального уравнения:

$$
x^{\prime}(\tau)=-x R_{0}(x)+(\lambda+\beta \psi) R_{1}(x)+(\lambda+\beta \varphi) R_{2}(x) .
$$

Таким образом, $x=x(\tau)$ является детерминированной функцией.

\section{3. Величины отклонения}

Обозначим правую часть дифференциального уравнения (15) как $A(x)$ :

$$
A(x)=-x R_{0}(x)+(\lambda+\beta \psi) R_{1}(x)+(\lambda+\beta \varphi) R_{2}(x) .
$$

Будем искать решение $H_{k}(y, s, \tau, \varepsilon)$ системы (14) в виде :

$$
H_{k}(y, s, \tau, \varepsilon)=Q_{k}(x, s) H(y, \tau)+\varepsilon h_{k}(y, s, \tau)+o(\varepsilon) .
$$

Прежде всего, отыщем вид функций $h_{k}(y, s, \tau)$. Подставим в систему (14) разложение (17), учтём (7) и запишем полученную систему, сократив на $\varepsilon$ все уравнения, в виде: 


$$
\begin{gathered}
-(\alpha+\lambda+x) h_{0}(y, s, \tau)+\mu_{1}(s) h_{1}(y, s, \tau)+\mu_{2}(s) h_{2}(y, s, \tau)+ \\
+\sum_{s_{1}=1}^{S} q_{s_{1} s} h_{0}\left(y, s_{1}, \tau\right)=Q_{0}(x, s) y H(y, \tau)- \\
-\left(x^{\prime}(\tau) Q_{0}(x, s)-\beta \mu_{1}(s) Q_{1}(x, s)-\beta \mu_{2}(s) Q_{2}(x, s)\right) \frac{\partial H(y, \tau)}{\partial y} \\
-\mu_{1}(s) h_{1}(y, s, \tau)+(\lambda+x) h_{0}(y, s, \tau)+\sum_{s_{1}=1}^{S} q_{s_{1}} h_{1}\left(y, s_{1}, \tau\right)= \\
=-Q_{0}(x, s) y H(y, \tau)-\left(\left(x^{\prime}(\tau)-\lambda\right) Q_{1}(x, s)+x Q_{0}(x, s)\right) \frac{\partial H(y, \tau)}{\partial y} \\
-\mu_{2} h_{2}(y, s, \tau)+\alpha h_{0}(y, s, \tau)+\sum_{s_{1}=1}^{S} q_{s_{1} s} h_{2}\left(y, s_{1}, \tau\right)= \\
=-\left(x^{\prime}(\tau)-\lambda\right) Q_{2}(x, s) \frac{\partial H(y, \tau)}{\partial y}
\end{gathered}
$$

Система (18) является неоднородной системой линейных алгебраических уравнений. Суммируя уравнения системы (18) и учитывая (15), нетрудно показать, что ранг собственной матрицы системы (18) совпадает с рангом её расширенной матрицы, следовательно, она имеет решение, определяемое с точностью до однопараметрического семейства векторов: $\left(Q_{0}(x, 1), \ldots, Q_{0}(x, S), Q_{1}(x, 1), \ldots, Q_{1}(x, S), Q_{2}(x, 1), \ldots, Q_{2}(x, S)\right)^{T} \cdot C$ , где $C$ - произвольная скалярная величина.

Будем искать решение системы (18) в следующем виде:

$$
h_{k}(y, s, \tau)=h_{k}^{(1)}(x, s) \frac{\partial H(y, \tau)}{\partial y}+h_{k}^{(2)}(x, s) y H(y, \tau) .
$$

Подставим (19) в (18) и представим систему в виде двух систем:

$$
\begin{gathered}
-(\alpha+\lambda+x) h_{0}^{(1)}(x, s)+\mu_{1}(s) h_{1}^{(1)}(x, s)+\mu_{2}(s) h_{2}^{(1)}(x, s)+\sum_{s_{1}=1}^{S} q_{s_{1} s} h_{0}^{(1)}\left(x, s_{1}\right)= \\
=-x^{\prime}(\tau) Q_{0}(x, s)+\beta \mu_{1}(s) Q_{1}(x, s)+\beta \mu_{2}(s) Q_{2}(x, s) \\
-\mu_{1}(s) h_{1}^{(1)}(x, s)+(\lambda+x) h_{0}^{(1)}(x, s)+\sum_{s_{1}=1}^{S} q_{s_{1} s} h_{1}^{(1)}\left(x, s_{1}\right)= \\
=-\left(x^{\prime}(\tau)-\lambda\right) Q_{1}(x, s)-x Q_{0}(x, s), \\
-\mu_{2}(s) h_{2}^{(1)}(x, s)+\alpha h_{1}^{(1)}(x, s)+\sum_{s_{1}=1}^{S} q_{s_{1} s} h_{2}^{(1)}\left(x, s_{1}\right)=-\left(x^{\prime}(\tau)-\lambda\right) Q_{2}(x, s)
\end{gathered}
$$

и

$$
\begin{gathered}
-(\alpha+\lambda+x) h_{0}^{(2)}(x, s)+\mu_{1}(s) h_{1}^{(2)}(x, s)+\mu_{2}(s) h_{2}^{(2)}(x, s)+ \\
+\sum_{s_{1}=1}^{S} q_{s_{1} s} h_{0}^{(2)}\left(x, s_{1}\right)=Q_{0}(x, s)
\end{gathered}
$$




$$
\begin{gathered}
-\mu_{1}(s) h_{1}^{(2)}(x, s)+(\lambda+x) h_{0}^{(2)}(x, s)+\sum_{s_{1}=1}^{S} q_{s_{1} s} h_{1}^{(2)}\left(x, s_{1}\right)=-Q_{0}(x, s), \\
-\mu_{2}(s) h_{2}^{(2)}(x, s)+\alpha h_{0}^{(2)}(x, s)+\sum_{s_{1}=1}^{S} q_{s_{1} s} h_{2}^{(2)}\left(x, s_{1}\right)=0 .
\end{gathered}
$$

Сопоставим результат дифференцирования системы (7) по $x$ и систему получим, получим, что решение $h_{k}^{(2)}(x, s)$ системы (21) имеет вид:

$$
h_{k}^{(2)}(x, s)=\frac{\partial Q_{k}(x, s)}{\partial x} .
$$

С учётом (22) и (19) разложение (17) примет вид:

$$
H_{k}(y, s, \tau, \varepsilon)=Q_{k}(x, s) H(y, \tau)+\varepsilon h_{k}^{(1)}(x, s) \frac{\partial H(y, \tau)}{\partial y}+\varepsilon y H(y, \tau) \frac{\partial Q_{k}(x, s)}{\partial x}+o(\varepsilon) \text {. }
$$

Далее найдём вид функции $H(y, \tau)$. Для этого функции в правой части системы (3) разложим в ряд по приращениям аргумента $y$ с точностью до $o\left(\varepsilon^{2}\right)$, получим:

$$
\begin{aligned}
& \varepsilon^{2} \frac{\partial H_{0}(y, s, \tau, \varepsilon)}{\partial \tau}-\varepsilon x^{\prime}(\tau) \frac{\partial H_{0}(y, s, \tau, \varepsilon)}{\partial y}+(\alpha+\lambda+x+\varepsilon y) H_{0}(y, s, \tau, \varepsilon)= \\
& =\mu_{1}(s) H_{1}(y, s, \tau, \varepsilon)+\mu_{2}(s) H_{2}(y, s, \tau, \varepsilon)-\beta \mu_{1}(s) \varepsilon \frac{\partial H_{1}(y, s, \tau, \varepsilon)}{\partial y}+ \\
& +\beta \mu_{1}(s) \frac{\varepsilon^{2}}{2} \frac{\partial H_{1}^{2}(y, s, \tau, \varepsilon)}{\partial y^{2}}-\beta \mu_{2}(s) \varepsilon \frac{\partial H_{2}(y, s, \tau, \varepsilon)}{\partial y}+ \\
& +\beta \mu_{2}(s) \frac{\varepsilon^{2}}{2} \frac{\partial H_{2}^{2}(y, s, \tau, \varepsilon)}{\partial y^{2}}+\sum_{s_{1}=1}^{S} q_{s_{1} s} H_{0}\left(y, s_{1}, \tau, \varepsilon\right)+o\left(\varepsilon^{2}\right), \\
& \varepsilon^{2} \frac{\partial H_{1}(y, s, \tau, \varepsilon)}{\partial \tau}-\varepsilon x^{\prime}(\tau) \frac{\partial H_{1}(y, s, \tau, \varepsilon)}{\partial y}+\left(\lambda+\mu_{1}(s)\right) H_{1}(y, s, \tau, \varepsilon)= \\
& =\lambda H_{1}(y, s, \tau, \varepsilon)-\varepsilon \lambda \frac{\partial H_{1}(y, s, \tau, \varepsilon)}{\partial y}+\lambda \frac{\varepsilon^{2}}{2} \frac{\partial^{2} H_{1}(y, s, \tau, \varepsilon)}{\partial y^{2}}+ \\
& +(\lambda+x+\varepsilon y) H_{0}(y, s, \tau, \varepsilon)+\varepsilon \frac{\partial}{\partial y}\left\{(x+\varepsilon y) H_{0}(y, s, \tau, \varepsilon)\right\}+ \\
& +x \frac{\varepsilon^{2}}{2} \frac{\partial^{2} H_{0}(y, s, \tau, \varepsilon)}{\partial y^{2}}+\sum_{s_{1}=1}^{S} q_{s_{1} s} H_{1}\left(y, s_{1}, \tau, \varepsilon\right)+o\left(\varepsilon^{2}\right), \\
& \varepsilon^{2} \frac{\partial H_{2}(y, s, \tau, \varepsilon)}{\partial \tau}-\varepsilon x^{\prime}(\tau) \frac{\partial H_{2}(y, s, \tau, \varepsilon)}{\partial y}+\left(\lambda+\mu_{2}(s)\right) H_{2}(y, s, \tau, \varepsilon)= \\
& =\alpha H_{0}(y, s, \tau, \varepsilon)+\lambda H_{2}(y, s, \tau, \varepsilon)+\lambda \varepsilon \frac{\partial H_{2}(y, s, \tau, \varepsilon)}{\partial y}+ \\
& +\lambda \frac{\varepsilon^{2}}{2} \frac{\partial^{2} H_{2}(y, s, \tau, \varepsilon)}{\partial y^{2}}+\sum_{s_{1}=1}^{S} q_{s_{1} s} H_{2}\left(y, s_{1}, \tau, \varepsilon\right)+o\left(\varepsilon^{2}\right) .
\end{aligned}
$$


Сложим уравнения системы (24) по $k$, подставим в полученную систему разложение функций $H_{k}(y, s, \tau, \varepsilon)$ в виде (23), учтём обозначение (9), получим:

$$
\begin{gathered}
\varepsilon^{2} r(s) \frac{\partial H(y, \tau)}{\partial \tau}-\varepsilon x^{\prime}(\tau) r(s) \frac{\partial H(y, \tau)}{\partial y}-\varepsilon^{2} x^{\prime}(\tau) \frac{\partial}{\partial x}\left\{\sum_{k=0}^{2} Q_{k}(x, s)\right\} \frac{\partial\{y H(y, \tau)\}}{\partial y}- \\
-\varepsilon^{2} x^{\prime}(\tau) \sum_{k=0}^{2} h_{k}^{(1)}(x, s) \frac{\partial^{2} H(y, \tau)}{\partial y^{2}}=-\varepsilon\left(-x Q_{0}(x, s)+\left(\lambda+\beta \mu_{1}(s)\right) Q_{1}(x, s)+\right. \\
\left.+\left(\lambda+\beta \mu_{2}(s)\right) Q_{2}(x, s)\right) \frac{\partial H(y, \tau)}{\partial y}-\varepsilon^{2}\left(-Q_{0}(x, s)-x \frac{\partial Q_{0}(x, s)}{\partial x}+\right. \\
\left.+\left(\lambda+\beta \mu_{1}(s)\right) \frac{\partial Q_{1}(x, s)}{\partial x}+\left(\lambda+\beta \mu_{2}(s)\right) \frac{\partial Q_{2}(x, s)}{\partial x}\right) \frac{\partial\{y H(y, \tau)\}}{\partial y}+ \\
+\frac{\varepsilon^{2}}{2}\left[x Q_{0}(x, s)+\left(\lambda+\beta \mu_{1}(s)\right) Q_{1}(x, s)+\left(\lambda+\beta \mu_{2}(s)\right) Q_{2}(x, s)+\right. \\
\left.+2\left(x h_{0}^{(1)}(x, s)-\left(\lambda+\beta \mu_{1}(s)\right) h_{1}^{(1)}(x, s)-\left(\lambda+\beta \mu_{2}(s)\right) h_{2}^{(1)}(x, s)\right)\right] \frac{\partial^{2} H(y, \tau)}{\partial y^{2}}+ \\
+\sum_{k=0}^{2} \sum_{s_{1}=1}^{S} q_{s_{1} s} H_{k}\left(y, s_{1}, \tau, \varepsilon\right)+o\left(\varepsilon^{2}\right) .
\end{gathered}
$$

Просуммируем уравнения системы (25) по $s$, учтём (9) и (10), также обозначим:

$$
\begin{array}{r}
\sum_{s=1}^{S} h_{k}^{(1)}(x, s)=h_{k}^{(1)}(x), \sum_{k=0}^{2} h_{k}^{(1)}(x)=h^{(1)}(x), \\
\sum_{s=1}^{S} \mu_{k}(s) h_{k}^{(1)}(x, s)=\eta_{k} h_{k}^{(1)}(x), k=1,2,
\end{array}
$$

учтём (1), получим:

$$
\begin{gathered}
\varepsilon^{2} \frac{\partial H(y, \tau)}{\partial \tau}-\varepsilon x^{\prime}(\tau) \frac{\partial H(y, \tau)}{\partial y}-\varepsilon^{2} x^{\prime}(\tau) h^{(1)}(x) \frac{\partial^{2} H(y, \tau)}{\partial y^{2}}= \\
=-\varepsilon\left(-x R_{0}(x)+(\lambda+\beta \psi) R_{1}(x)+(\lambda+\beta \varphi) R_{2}(x)\right) \frac{\partial H(y, \tau)}{\partial y}- \\
-\varepsilon^{2}\left(-R_{0}(x)-x \frac{\partial R_{0}(x)}{\partial x}+(\lambda+\beta \psi) \frac{\partial R_{1}(x)}{\partial x}+(\lambda+\beta \varphi) \frac{\partial R_{2}(x)}{\partial x}\right) \frac{\partial\{y H(y, \tau)\}}{\partial y}+ \\
+\frac{\varepsilon^{2}}{2}\left[x R_{0}(x)+(\lambda+\beta \psi) R_{1}(x)+(\lambda+\beta \varphi) R_{2}(x)+\right. \\
\left.+2\left(x h_{0}^{(1)}(x)-\left(\lambda+\beta \eta_{1}\right) h_{1}^{(1)}(x)-\left(\lambda+\beta \eta_{2}\right) h_{2}^{(1)}(x)\right)\right] \frac{\partial^{2} H(y, \tau)}{\partial y^{2}}+o\left(\varepsilon^{2}\right) .
\end{gathered}
$$

В силу дифференциального уравнения (15) уничтожим слагаемые порядка $O(\varepsilon)$, поделим обе части полученного уравнения на $\varepsilon^{2}$, выполним преобразования, будем иметь: 


$$
\begin{gathered}
\frac{\partial H(y, \tau)}{\partial \tau}=-\left(-R_{0}(x)-x \frac{\partial R_{0}(x)}{\partial x}+(\lambda+\beta \psi) \frac{\partial R_{1}(x)}{\partial x}+(\lambda+\beta \varphi) \frac{\partial R_{2}(x)}{\partial x}\right) \times \\
\times \frac{\partial\{y H(y, \tau)\}}{\partial y}+\frac{1}{2}\left(\left(1+2 h^{(1)}(x)\right) x^{\prime}(\tau)+2 x\left(R_{0}(x)+h_{0}^{(1)}(x)\right)-2 \lambda\left(h_{1}^{(1)}(x)+\right.\right. \\
\left.+h_{2}^{(1)}(x)\right)-2 \beta\left(\eta_{1} h_{1}^{(1)}(x)+\eta_{2} h_{2}^{(1)}(x)\right) \frac{\partial^{2} H(y, \tau)}{\partial y^{2}} .
\end{gathered}
$$

Получили уравнение Фоккера - Планка для плотности распределения вероятностей $H(y, \tau)$ значений диффузионного процесса авторегрессии $y(\tau)$. Коэффициент переноса уравнения (28) есть производная по $x$ от правой части уравнения (15), то есть, в силу обозначения (16), - $A_{x}^{\prime}(x)$.

Коэффициент диффузии обозначим следующим образом:

$$
\begin{gathered}
B^{2}(x)=\left(1+2 h^{(1)}(x)\right) x^{\prime}(\tau)+2 x\left(R_{0}(x)+h_{0}^{(1)}(x)\right)-2 \lambda\left(h_{1}^{(1)}(x)+h_{2}^{(1)}(x)\right)- \\
-2 \beta\left(\eta_{1} h_{1}^{(1)}(x)+\eta_{2} h_{2}^{(1)}(x)\right) .
\end{gathered}
$$

Из (28) с учётом (29) следует, что $H(y, \tau)$ - есть плотность вероятностей диффузионного процесса $y(\tau)$, удовлетворяющего стохастическому дифференциальному уравнению:

$$
d y(\tau)=A_{x}^{\prime}(x) y(\tau) d \tau+B(x) d w(\tau),
$$

где $w(\tau)$ является стандартным процессом Винера [14].

\section{Заключение}

В работе предложена модель RQ-систем с вызываемыми и возвратными заявками в случайной среде, моделируемой цепью Маркова с непрерывным временем. Асимптотическим методом [13] получено дифференциальное уравнение (15), определяющее среднее $x=x(\tau)$ нормированного числа заявок на орбите. Представлено распределение $R_{k}(x), k=0,1,2$ вероятностей состояний $k$ прибора в виде (13). Показано, что процесс $y(\tau)$, характеризующий изменение величин отклонения от среднего, является диффузионным процессом авторегрессии и определяется стохастическим дифференциальным уравнением (30). Полученные результаты могут быть использованы при анализе и планировании деятельности call-центров.

\section{$* * *$}

1. Artalejo J.R., Gomez-Corral A. Retrial Queuing Systems: A Computational Approach. Berlin: Springer, Heidelberg, 2008. $318 \mathrm{p}$.

2. $\quad$ Falin G.I., Templeton J.G.C. Retrial Queues. London: Chapman and Hall, 1997. 328 c.

3. Коротаев И. А. Системы массового обслуживания с переменными параметрами. Томск: Изд-во Том. ун-та, 1991. $167 \mathrm{c}$.

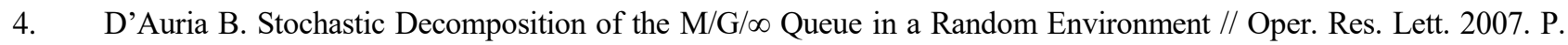
805-812.

5. Kim C.S., Klimenok V., Mushko V., Dudin A. The BMAP/P H/N Retrial Queuing System Operating in Markovian Random Environment // Comput. Oper. Res. 2010. Vol. 37. \# 7. P. 1228-1237.

6. O'Cinneide C.A., Purdue P. The M/M/o Queue in a Random Environment // J. Appl. Prob. 1986. V. 23. P. 175-184.

7. Sztrick J. On the Heterogeneous M/G/N Blocking System in a Random Environment // Journal of Operations Research Society. 1987. Vol. 38. \# 1. P. 57-63.

8. Дудин А.Н., Назаров А.А. Система обслуживания ММАР $/ \mathrm{M} / \mathrm{R} / 0$ с резервированием приборов, функционирующая в случайной среде // Проблемы передачи информации. 2015. Том 51. Вып. 3. С. 93-104.

9. D'Auria B. M/M/o Queues in semi-Markovian Random Environment // Queuing Syst. 2008. Vol. 58. P. 221-237.

10. Fralix B.H., Adan I.J.B.F. An Infinite-server Queue Influenced by a semi-Markovian Environment // Queuing Syst. 2009. P. 65-84.

11. Вавилов B.А. Анализ функционирующих в полумарковской среде RQ-систем с возвратом заявок // Кибернетика и программирование. 2019. № 1. С. 18-36.

12. Боровков А.А. Асимптотические методы в теории массового обслуживания. М.: Наука, 1980. 210 c. 
13. Назаров А.А., Моисеева С.П. Метод асимптотического анализа в теории массового обслуживания. Томск: Изд-во НТЛ, 2006. 112 с.

14. Карлин С. Основы теории случайных процессов. М.: Мир, 1971. 536 с.

15. Эльсгольц Л.Е. Дифференциальные уравнения и вариационное исчисление. М.: Наука, 1969. 424 с.

\section{Зонин Г.Д. \\ Гибридные системы в сферах производства}

Российский технологический университет МИРЭА

(Россия, Москва)

doi: 10.18411/trnio-11-2021-38

\section{Аннотация}

В данной статье рассматриваются основы гибридных систем в различных сферах деятельности. Искусственный интеллект вышел на новый уровень в IT сфере. Многие компании прибегают к возможностям машинного кода, тем самым обеспечив себе качественное и безопасное выполнение работы в различных отраслях деятельности. Способность к самообучению, скорость выполнения, возможность использования на разных платформах - все это открывает новые горизонты в информационной индустрии для всего человечества.

Ключевые слова: гибридные системы, искусственный интеллект, автоматизация, инновации, искусственная нейронная сеть, машинное зрение.

\section{Abstract}

This article discusses the basics of hybrid systems in various fields of activity. Artificial intelligence has reached a new level in the IT sphere. Many companies resort to the capabilities of machine code, thereby ensuring high-quality and safe performance of work in various fields of activity. The ability to self-study, the speed of execution, the ability to use it on different platforms - all this opens up new horizons in the information industry for all mankind.

Keywords: hybrid systems, artificial intelligence, automation, innovation, artificial neural network, machine vision.

Двадцать первый век - век технологий и инноваций. Именно в это время область использования искусственного интеллекта вышла на новый уровень. Одним из разделов в данной сфере являются автоматизированные системы.

Автоматизация - это одна из возможностей научно-технического прогресса, которая позволяет человеку избежать лишних затрат времени, сил, нервов, но в то же время, улучшить качество выполняемых задач. Данный термин получил широкую область использования после 1947 года. В этот промежуток времени американская автомобилестроительная компания создала отдел автоматизации, тем самым позволив индустрии выйти на новый уровень.

Автоматизация является независимой частью гибридной интеллектуальной системы, которая в свою очередь использует более одного метода имитации интеллектуальной деятельности человека для решения той или иной задачи. Реализация гибридных систем осуществляется путем использования нескольких методов по реализации задач, связанных с управлением и проектированием. Так, например, системы безопасности является неотъемлемой составляющей любого общественного заведения. Чтобы следить за безопасностью или же просто отслеживать действия физических лиц, необходимы гибридные системы, позволяющие распознавать окружающие лица и записывать их в базы данных для дальнейшего взаимодействия с ними.

Рассмотрим гибридную систему на примере системы безопасности московского метрополитена. Каждые камеры оснащены датчиками передвижения. Видеоизображение 